\title{
Black-box model identification for a continuously variable, electro-hydraulic semi-active damper
}

\author{
M. Witters, J. Swevers \\ Division PMA, Department of Mechanical Engineering, Katholieke Universiteit Leuven, \\ Celestijnenlaan 300B, B-3001 Heverlee, Belgium (Tel: + 32163224 80).
}

\begin{abstract}
This paper discusses the black-box identification of a continuously variable, electro-hydralic semi-active damper for a passenger car. A neural network based output error model structure (NNOE) is selected to describe the complex nonlinear damper dynamics. This model structure is able to accurately and efficiently describe the dynamic damper behaviour, and is appropriate for full vehicle simulation. The identification procedure includes optimal experiment design, regression vector selection and model parameter estimation. The damper excitation signals are optimized multisines that yield uniform coverage of the achievable working range of the damper. A state of the art iterative procedure is used to concurrently estimate the model parameters and select an optimal set regression vector elements. Experimental validation of the proposed procedure shows that models identified from the data measured using the optimized excitations are considerably more accurate than those identified from data obtained using conventional random phase multisine excitations.
\end{abstract}

Key words:

Semi-active damper, vehicle suspension, identification, experiment design, nonlinear system, neural network model

\section{Introduction}

Nowadays, most luxury cars are equipped with semi-active suspension systems and more recently they become also available for mid class vehicles. It is expected that their market share will continue to grow rapidly and their performance will improve too [1]. Two types of semi-active dampers are currently applied in car suspensions: magneto-rheological $(M R)$ dampers and continuously variable, electro-hydraulic semi-active dampers (CVEHSA). When a magnetic field is applied to a magneto-rheological oil, the micron-sized, polarizable particles suspended in the fluid tend to form chain-like structures, which modifies the

Email address: jan.swevers@mech.kuleuven.be (M. Witters, J. Swevers)

$U R L$ : www. mech.kuleuven. be (M. Witters, J. Swevers)

Preprint submitted to Mechanical Systems and Signal Processing

March 20, 2009 
yield stress of the fluid. In the piston of a MR-damper, a solenoid is mounted that allows to apply a magnetic field over the orifice through which the oil flows when the damper is moving. Hence, the damping characteristic can be altered by controlling the current applied to the solenoid. The CVEHSA-damper is equipped with a servo-valve that allows to vary continuously the orifice through which the oil flows when the damper is moving.

Semi-active dampers exhibit one major inconvenience: their inherent nonlinear dynamic behaviour which complicates the design of a controller for the suspension system. Swevers et al. [3] have developed a parameterized, modelfree control structure for a passenger car equipped with an electro-hydraulic semi-active suspension system, that can be tuned based on the directions of a test-pilot. However, the road tests required to tune the controller are time consuming and costly. A reliable full vehicle simulation model would allow the car manufacturers to overcome this drawback. This requires a model for the semi-active damper dynamics that accurately describes the damper force as a function of the rattle velocity and current in the frequency band up to about $30 \mathrm{~Hz}[2]$.

In the past, a lot of effort has been invested in developing models and experimental identification methods for passive hydraulic dampers and magnetorheological (MR) dampers, ranging from complicated physical models ([4]) to nonlinear black-box models ([5], [6], [7]). These black-box techniques have shown to yield the most accurate descriptions of the damper dynamics. Blackbox models use general mathematical approximating functions to describe the systems input-output relations. Hence, the most important advantages of these modelling techniques are the computational efficiency, both in model identification as in simulation, and the limited physical insights required to develop the model. However, there remain two challenging steps in the identification of black-box models for highly non-linear systems:

- the selection of the most suitable set of regression variables,

- the design of excitation signals that yield experimental data that uniformly covers the entire working range of the system.

The identification procedure presented in this paper uses a state of the art technique based on simulation error minimization [8] to select an optimal set of regression variables. The authors have shown in previous work [9] that this method works well for the identification of a neural network based output error model for an electro-hydraulic semi-active damper. With respect to this earlier work the procedure is extended with an optimal experiment design method that selects the phases of a multisine excitation such that the total working range of the damper is uniformly covered. In addition, this papers presents an elaborate experimental validation of the effectiveness of the experiment design method and the regression vector selection procedure.

The paper is organized as follows. Section 2 describes the considered semiactive damper and reviews briefly some literature on the modelling of passive and magneto-rheological dampers. Section 3 gives a short overview of the used 
concepts of black-box modelling: it introduces the considered model structure, followed by a discussion on the identification procedure that includes the model structure selection algorithm and the parameter estimation method. Section 4 describes the test setup and the optimal experiment design procedure. In section 5 the effectiveness of the presented methods are experimentally verified by identifying and validating a simulation model for a semi-active damper. Section 6 presents the conclusions and gives some suggestions for improvements.

\section{Semi-active damper characteristics}

The first part of this section discusses the structure of CVEHSA-dampers by comparing it to classical passive dampers, from which the design has been derived. The next part shows the characteristics of the damper and illustrates the highly nonlinear dynamic behaviour, while the last part reviews some models of passive and magneto-rheological dampers. These dampers exhibit a dynamic behaviour similar to that of the CVEHSA-damper. Hence, these models are useful background information for the development of a model for the CVEHSAdampers.

\subsection{Working principle}

A classic passive damper consists of a cylinder filled with oil and a rod connected to a piston which contains a calibrated restriction called the piston valve (see figure 1 left). The change in volume caused by the rod moving in or out of the cylinder is compensated for by oil flowing in or out of the accumulator (accu) through the base valve. The pressure drop over both the base valve and the piston valve results in a damping force acting on the piston.

The structure of the considered semi-active damper hardware (see figure 1 right) and that of a passive damper are similar except for the piston and base valve which are each replaced by a check valve. In addition, a current-controlled, continuously variable semi-active valve (cvsa) connects the upper and lower damper chambers. The current to this valve is limited by the control system between $i^{-}=0.3 \mathrm{~A}$ and $i^{+}=1.6 \mathrm{~A}$, which corresponds to the least and most restrictive settings of the valve (i.e. open and closed), respectively. When the rod moves upwards (positive rattle velocity, i.e. rebound of the suspension), the piston check valve closes and oil flows through the cvsa-valve. Because the volume of the rod inside the cylinder reduces, oil is forced from the accumulator into the cylinder through the base check valve.

When the rod moves downwards (negative rattle velocity, i.e. compression of the suspension), the piston check valve opens. Because the volume of the rod inside the cylinder increases, the base check valve closes and oil flows from the cylinder into the accumulator through the cvsa-valve. A low/high current to the cvsa-valve corresponds to a small/large restriction yielding a low/high damping ratio. 


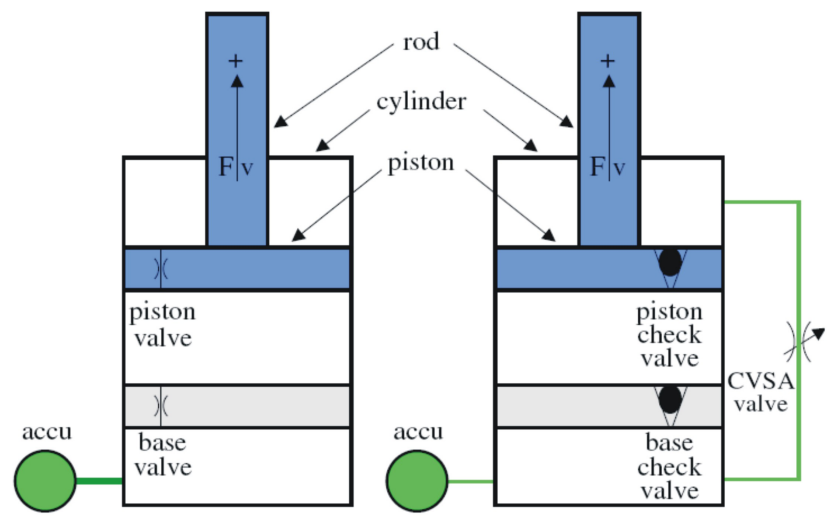

Figure 1: Working principle of a passive and a semi-active damper.

\subsection{Continuously variable damping characteristic}

To illustrate the characteristics of the CVEHSA-damper, figures 2 and 3 show the damper forces measured while enforcing a sinusoidal displacement of the piston with amplitudes of 5 and $2 \mathrm{~cm}$ at 1.5 and $5 \mathrm{~Hz}$ respectively. During the measurements, the current to the cvsa-valve is kept constant at four different levels $(I=0.3,0.7,1.2$ and $1.6 \mathrm{~A})$. The measurements are performed on a hydraulic test rig, which is discussed in section 4.

Remark that the four graphs of the figures 2 and 3 each have a different scale of the y-axis in order to show clearly the shape of the measured force curves.

These figures illustrate the highly complex nonlinear dynamic behaviour of the damper. The damper response depends on the amplitude and frequency of excitation; it exhibits hysteresis and saturation at large velocities. In addition to these nonlinear effects, the response also depends on the dynamics of the csva-valve.

\subsection{Damper models}

The CVEHSA-damper is an evolution of the conventional passive damper, as explained in section 2.1. As a consequence, the two devices exhibit a similar dynamic behaviour, including hysteresis and saturation. Results obtained in the modelling of passive dampers can thus be an interesting source of information for the development of a black-box semi-active damper model.

Duym et al. developed a complex, continuous-time physical model for a conventional passive damper, see [4] and the references therein. However, the identification of the model parameters is cumbersome and simulations of the model are slow. This motivates the application of nonlinear black-box modelling techniques to describe the damper dynamics. Patel and Dunne [5] compared a neural network based $N A R X$ model, using damper velocity and temperature as inputs, to the white-box model of a passive damper proposed by Duym [4]. They show 

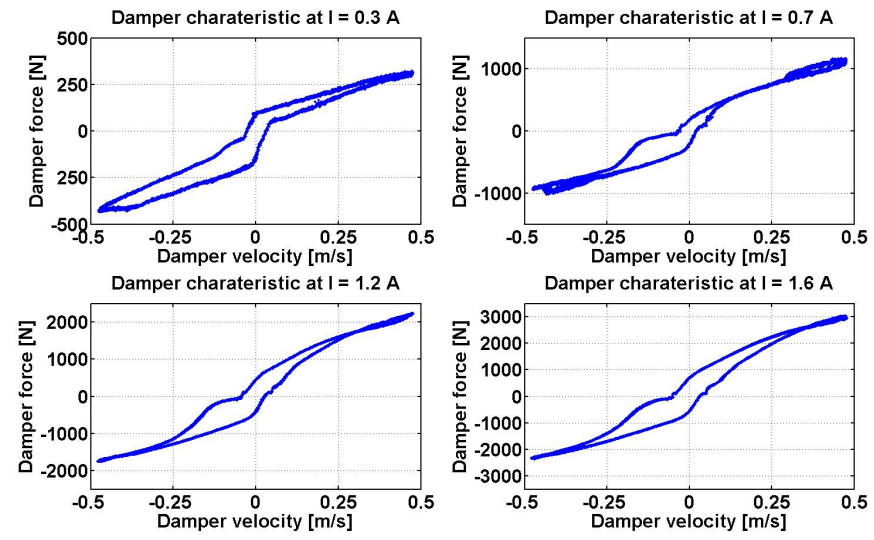

Figure 2: Damper response for a sinusoidal displacement at $1.5 \mathrm{~Hz}, 5 \mathrm{~cm}$ amplitude and constant current excitation $(I=0.3,0.7,1.2$ and $1.6 \mathrm{~A})$
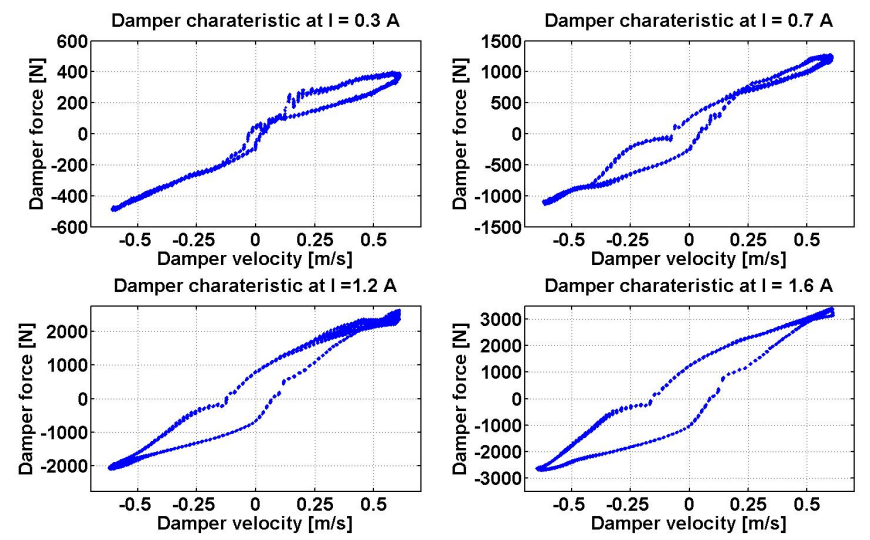

Figure 3: Damper response for a sinusoidal displacement at $5 \mathrm{~Hz}, 2 \mathrm{~cm}$ amplitude and constant current excitation $(I=0.3,0.7,1.2$ and $1.6 A)$ 
that the identification of the presented black-box model is much easier then that of the white-box model, while obtaining the same level of accuracy with both models [5]. Duym also investigated the use of static state-force maps to model passive dampers. He reported that models which include the damper velocity and acceleration as inputs yield a higher accuracy than those using the damper displacement and velocity.

A lot of effort has been invested in the modelling of magneto-rheological dampers. Spencer et al. [12] proposed a grey-box approach using the Bouc-Wen model [13] to describe the hysteresis of the device. More recently, nonlinear blackbox modelling techniques have been applied to describe the dynamics of MR dampers. Leva and Piroddi developed a polynomial $N A R X$ model [8], while Savaresi used a neural network based NARX model and demonstrated that the proposed model outperforms the grey-box model developed by Spencer [7]. Savaresi included the damper displacement and velocity in the regression vector. This paper discusses the identification of a discrete time, black-box simulation model using a neural network based output error model structure (NNOE) [14] for a continuously variable, electro-hydraulic semi-active damper. The neural network based model structure has demonstrated to be able to describe accurately the dynamic behaviour of a wide class of complex nonlinear systems (see e.g. [14], [7], [15], ...). An output error structure is selected such that the model is suitable to be implemented in a full vehicle simulation model.

The next section discusses briefly the applied black-box model structure selection and identification.

\section{Black-box modelling}

This section discusses the identification of the CVEHSA-damper using the NNOE model structure. First, some general concepts of NNOE structure are reviewed, then the model structure and regression variable selection and parameters estimation steps of the identification procedure are discussed.

\subsection{NNOE model structure}

The identification of a discrete time black-box model for a dynamic system [16] is the problem of finding a parameterized relation, $g$, between the past observed inputs $u^{t_{n-1}}=\left[u\left(t_{n-1-n_{k}}\right), \ldots, u\left(t_{n-n_{k}-n_{b}}\right)\right]$ and outputs $y^{t_{n-1}}=$ $\left[y\left(t_{n-1}\right), \ldots, y\left(t_{n-n_{a}}\right)\right]$ and the current output, $y\left(t_{n}\right)$ :

$$
y\left(t_{n}\right)=g\left(u^{t_{n-1}}, y^{t_{n-1}}, \theta\right)+\nu\left(t_{n}\right)
$$

where $\theta$ represents the parameter vector and $\nu$ an additive noise term. $n_{k}$ refers to a pure delay in the system, while $n_{a}$ and $n_{b}$ are the number of elements of the output and input variables selected to describe this relation $g$.

Sjöberg et al. [16] have shown that the relation $g$ can be split into two mappings: a first one $\phi$ maps the past inputs and outputs into a regression vector $\varphi(t)$ and a second $\psi$ maps the regression vector to the output space:

$$
\widehat{y}\left(t_{n}\right)=\psi\left(\varphi\left(t_{n}\right), \theta\right),
$$


where $\varphi\left(t_{n}\right)=\phi\left(u^{t_{n-1}}, y^{t_{n-1}}\right), \theta$ is the parameter vector of the model and $\widehat{y}\left(t_{n}\right)$ the model output.

Since the aim is to develop a simulation model for a semi-active damper, no measured outputs are allowed in the regression vector. Therefore, an outputerror structure is adopted for the regression vector, which only depends on the past inputs and model outputs $\widehat{y}$, up to time $t_{n-1}$ :

$$
\varphi\left(t_{n}\right)=\left[\begin{array}{llllll}
\widehat{y}\left(t_{n-1}\right) & \ldots & \widehat{y}\left(t_{n-n_{a}}\right) & u\left(t_{n-1-n_{k}}\right) & \ldots & u\left(t_{n-n_{k}-n_{b}}\right)
\end{array}\right]
$$

For the second mapping, a neural network is applied. A neural network can be defined as a system of neurons that are connected into a network by a set of weights [14]. The principle of a neuron $j$ is illustrated in figure 4: it is a processing element that weights $\left(w_{j i}\right)$ its $m$ inputs $r_{i}$, sums them and then returns the corresponding value $z_{j}$ of its activation function $f_{j}$. The input 1 is introduced to allow a bias of the weighted sum, $w_{j 0}$.

In this papar two types of activation functions will be used:

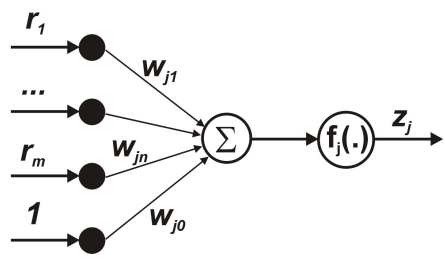

Figure 4: A neuron: $z_{j}=f_{j}\left(\sum_{i=1}^{m} w_{j i} r_{i}+w_{j 0}\right)$

- sigmoidal neurons which are characterized with following activation function:

$$
z_{j}=1-\frac{2}{e^{2\left(\sum_{i=1}^{m} w_{j i} r_{i}+w_{j 0}\right)}+1},
$$

- linear neurons that return the weighted sum of their inputs:

$$
z_{j}=\sum_{i=1}^{m} w_{j i} r_{i}+w_{j 0}
$$

Often, the nodes are arranged in layers and the inputs of the neurons in a layer are the outputs of the neurons in the previous layer. Such a network is referred to a multilayer perceptron. The last layer is generally referred to as the output layer, the other layers are called hidden layers. For the considered application, a network with one hidden layer of sigmoid neurons and a linear output layer is selected. It is demonstrated [17] that this type of networks is able to approximate any kind of continuous function given that the hidden layer 
contains a sufficient number of nodes.

The overall equation of a NNOE model is given by:

$$
\widehat{y}\left(t_{n}\right)=\psi\left(\varphi\left(t_{n}\right), \theta\right)=\sum_{j=1}^{n_{h}} W_{j} f_{j}\left(\sum_{i=1}^{n_{\varphi}} w_{j i} \varphi_{i}+w_{j 0}\right)+W_{0},
$$

where $n_{h}$ is the number of neurons in the hidden layer and $n_{\varphi}$ is the number of variables in the regression vector. The weights of the output node are represented by $W_{j}$, while $W_{0}$ indicates the bias of the linear output node. The parameter $w_{j i}$ is the weight applied by the $j^{t h}$ hidden node to the $i_{t h}$ regressor. The weight $w_{j 0}$ is the bias of the $j^{t h}$ hidden node.

The total black-box model structure is represented schematically in figure 5 . The TDL-rectangles represent a tapered delay line applied to the inputs and the model output. The tapered delay line can be interpreted as a memory function that stores values of past inputs and model outputs to assemble the regression vector $\varphi\left(t_{n}\right)$. The part in front of the hidden layer corresponds to the first mapping of the black-box model, while the second mapping, the neural network, is represented in the last part of the scheme. Additional unitary inputs have been added to the hidden layer and the output layer in order to introduce the bias of the activation functions. The arrows connecting regressor variables with the hidden nodes or the outputs of the hidden nodes with the output node represent the weights of the neural network.

The extension of the model structure to multiple input, multiple output sys-

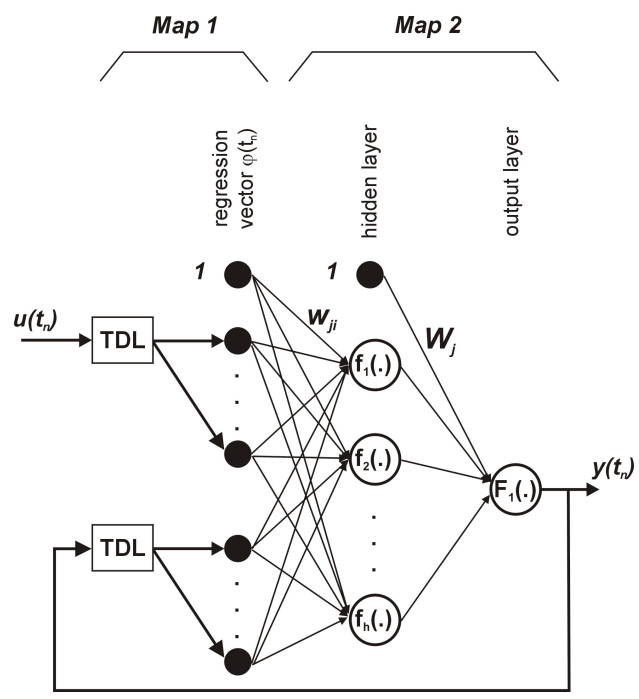

Figure 5: Schematic representation of the NNOE model structure.

tems $(M I M O)$ is straightforward: the regression vector will consist of delayed values of the different input and output variables. The tapered delay line can be 
chosen differently for each variable. The semi-active damper is an example of a multiple input system: in section 5 models are identified that use both damper displacement, velocity and acceleration and current as input variables.

The following two sections discuss the selection of the regression variables and the parameter estimation.

\subsection{Selection of the regression vector}

One of the most difficult aspects of black-box modelling is the selection of the regressor variables: which variables and how many elements of each variable are necessary and sufficient to describe the systems dynamics. A possible source of information is physical insight in the modelled system. From the literature review presented in section 2.3, the damper displacement, velocity, acceleration and of course the control current are selected as possible inputs.

Several algorithms have been proposed to select the optimal regression vector automatically (see [8] and the references therein), but none of them guarantees an optimal solution. In general, these algorithms proceed iteratively: at each iteration step a regression variable is added according to some criterion, followed by an estimation of the model parameters. The algorithm continues until a desired accuracy is reached or no improvement can be realized by adding more regression variables.

Here, the regressor selection procedure proposed by Piroddi and Spinelli [8], is applied, mainly because of its successful application to identify a polynomial $N A R X$ model for a MR damper [6]. Figure 6 shows a flow diagram of the iterative identification procedure. The different steps of this procedure are now briefly discussed.

Initialization: At the start of the procedure, the user has to provide the

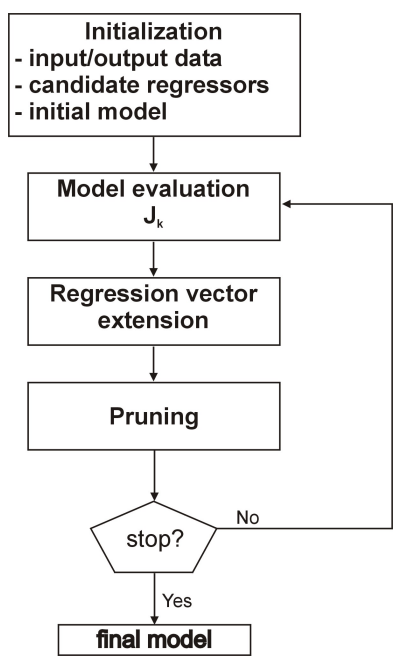

Figure 6: Flow diagram of the identification procedure.

measured input/output data, an initial model structure and a set of candidate 
regression variables. Limits on the number of delays $\left(n_{k}\right)$ and elements $\left(n_{a}\right.$ and $n_{b}$ ) for each variable that may be included in the regression vector, also have to be specified. Before proceeding, the parameters of the initial model are estimated.

Model evaluation: At the beginning of each iteration step, the performance of the model from the previous iteration or of the initial model if the identification procedure is in its first iteration, is evaluated by calculating the mean square simulation error $\left(J_{k}\right)$ :

$$
J_{k}=\sum_{n=1}^{N}\left(y\left(t_{n}\right)-\widehat{y}\left(t_{n}\right)\right)^{2} / N .
$$

Regression vector extension: This part of the procedure selects the additional regression variable that yields the largest model performance improvement. In order to accomplish this, for each possible extension of the regression vector with one element, the model parameters are estimated and the mean square simulation error performance index is calculated. The variable that yields the largest reduction of this performance index is selected.

Pruning: First, the parameters of all submodels obtained by eliminating one of the regression variables are estimated and evaluated using the above mentioned performance index. Second, if the best reduced model yields a performance improvement with respect to the model of the previous iteration, it is selected as the new model. This pruning continues until all models with fewer regressors yield a worse performance than the model of the previous iteration step.

Stop: The identification procedure is stopped when no model structure can be found that yields a better performance or the performance index is below a certain threshold value.

The threshold value used in the stop criterion obviously determines the length of the regression vector: increasing this threshold value for the performance index yields a shorter regression vector.

When applied to the identification of a neural network based black-box model, the algorithm does not optimize the network size and number of hidden nodes each time a new regression vector is selected in the extension and pruning steps of the algorithm. The number of hidden nodes is fixed at the beginning. One can however include an additional loop in the algorithm to optimize the network size, but this will increase the computational cost considerably.

\subsection{Parameter estimation}

The parameters of the neural network are estimated using the methods implemented in the Neural Network Based System Identification Toolbox (NNSYSID) [18]. The basic concepts of the applied methods will be reviewed in the following. For a more detailed treatment of the matter, the interested reader is referred to [14].

The parameter estimation problem can be formalized in the following way: given a set of measurement data

$$
Z^{N}=\left\{\left[u\left(t_{n}\right), y\left(t_{n}\right)\right], n=1, \ldots, N\right\},
$$


the model parameter vector $\widehat{\theta}$ can be found by solving following optimization problem:

$$
\widehat{\theta}=\arg \min _{\theta}\left\{V_{N}\left(\theta, Z^{N}\right)\right\},
$$

where $V_{N}\left(\theta, Z^{N}\right)$ is the objective function, expressing the distance between the model output and the measured system output. The most commonly used objective function for this type of problems is following regularized least squares error criterion [16]:

$$
V_{N}\left(\theta, Z^{N}\right)=\frac{1}{2 N} \sum_{n=1}^{N}\left[y\left(t_{n}\right)-\widehat{y}\left(t_{n} \mid \theta\right)\right]^{2}+\frac{1}{2 N} \theta^{T} D \theta,
$$

where $\mathrm{D}$ is called the weight decay term and is often chosen as a diagonal matrix $D=\alpha I$, with $\alpha$ the weight decay. The regularization term in the objective function, $\frac{1}{2 N} \theta^{T} D \theta$, prevents overfitting of network parameters. The regularization has also a smoothing effect on the objective function and hence will allow the optimization algorithm to proceed faster.

The least squares problem (9) is solved numerically by the Levenberg-Marquardt algorithm. Details of this method can be found in general text books about numerical optimization, e.g. [19]. Remark that the calculation of the derivative of the objective function with respect to the network weights is not trivial, since the network includes a feedback loop (see figure 5). Nörgard [14] demonstrated that this derivative can be obtained by filtering the partial derivative with a time-varying filter. The method can have convergence problems when the filter has instable roots. However, in proximity of the optimum, this should not occur. This implies that the initialization of the network weights should be close to the optimum. Therefore, the NNSYSID toolbox initializes the weights of the $N N O E$-model with those of the corresponding $N N A R X$-model.

\section{Test setup and experiment design}

To identify a reliable system model, the applied excitation signals should resemble as much as possible the normal operational load of the system [20]. Therefore, broad band excitation signals are used for the identification of the semi-active damper. The spectra of these excitations are derived from measurements on a test vehicle equipped with the semi-active suspension system [3]. The applied excitations are periodic multisines signals since they allow an easy design and offer some advantages for the postprocessing of the measurement data.

To obtain a model with an equal accuracy over the entire working range of the damper, the samples should be spread uniformly over the working range. To achieve this, the phases of the multisine excitations are optimized according to a procedure developed by Duym et al. [10].

In the first part of this section, the hydraulic test rig is presented, while the second part discusses the experiment design. 


\subsection{Hydraulic test rig}

The measurements are performed on a position controlled hydraulic test rig at the Monroe European Technical Center of Tenneco Automotive: the semiactive damper is excited by a hydraulic actuator, imposing a predefined damper displacement. Figure $7(\mathrm{a})$ shows the semi-active damper mounted in the test rig. At the top, the damper is connected to a load cell that measures the generated damper force $F$. At the bottom, the damper is driven by the position controlled hydraulic actuator of the test rig. The relative displacement of the rod with respect to the cylinder, $x_{r}$, is measured with a build-in displacement sensor (lvdt), which corresponds to the damper displacement. Figure 7(b) shows a schematic representation of the test rig. The current applied to the cvsa-valve is also measured. From the tubes shown at the right side of figure 7(a), air is blown over the damper to keep the damper temperature constant.

The damper velocity $v_{r}$ and acceleration $a_{r}$ are not measured, but they can be calculated accurately in frequency domain based on the measured displacement when these signals are periodic. No leakage errors are introduced because of the periodicity. First, the displacement signal is transformed to the frequency domain using the discrete Fourier transform. Next, the spectrum is multiplied by a rectangular window eliminating all frequencies that contain noisy data only. The resulting spectrum is then multiplied by the continuous-time frequency domain representation of a differentiator at the selected frequencies. A transformation back into the time domain using the inverse DFT yields an estimate of the first time derivative of the original signal, that is, the damper velocity. The spectrum of the damper acceleration is obtained by applying this method analogously to the calculated velocity. When multiple periods are measured, the noise level can be reduced even further by averaging the spectra of the different periods [20].

The fastest component of the semi-active damper is the cvsa-valve, which has an estimated response time of about $10 \mathrm{~ms}$. Therefore, the generated damper force $F$, the realized displacement $x_{r}$ and the current $i$ are sampled 10 times faster, at $1000 \mathrm{~Hz}$. The same sampling frequency is used for the models.

\subsection{Optimal multisine excitation}

Literature and insights in the damper dynamics have shown that the current to the cvsa-valve and the damper velocity are the two variables that mostly influence the damper response. Hence, a two-dimensional input space is considered, which should be covered uniformly within the limitations of the damper. These limitations are the minimal and maximal current (resp. $0.3 A$ and 1.6 $A)$, the damper displacement $(+/-6 \mathrm{~cm})$ and a restricted velocity $(+/-1.5$ $\mathrm{m} / \mathrm{s}$ ).

For the excitations, periodic multisine signals are used [20]:

$$
x(t, \chi)=\sum_{k=-F}^{F}\left|U_{k}\right| e^{j 2 \pi f_{\max } k t / F+j \chi_{k}},
$$




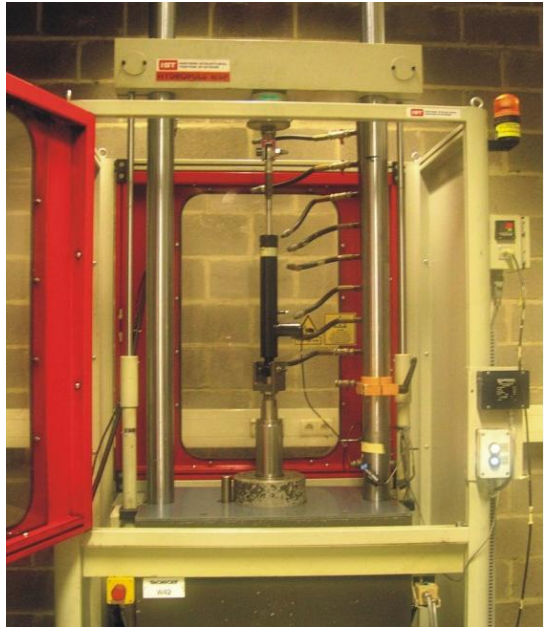

(a)

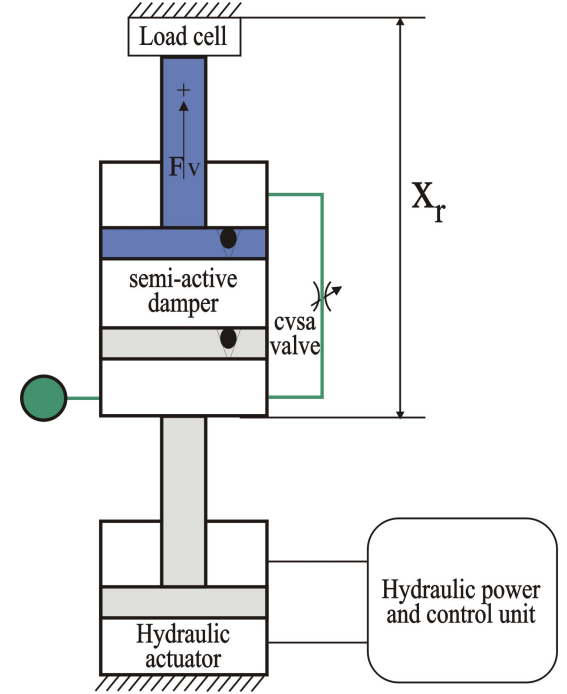

(b)

Figure 7: (a) Schenk hydraulic test rig (b) Schematic representation of the test rig.

with $f_{\max }$ the maximum frequency of the excitation signal, $F \in \mathbb{N}$ the number of excited frequency components and $\chi=\left[\chi_{1}, \ldots, \chi_{f}\right]^{T}$ the phases of these components.

The design of this multisine excitation signal involves the selection of:

- the frequency band of interest,

- the amplitude spectrum,

- the phases.

The maximum frequency of the displacement excitation signal is restricted to $30 \mathrm{~Hz}$, which corresponds to the bandwidth of the test rig actuator. This bandwidth is however sufficiently high since the heave, pitch and wheel hop resonances occur at lower frequencies, typically between 1 and $3 \mathrm{~Hz}$ for the modal resonances of the car body and between 10 and $20 \mathrm{~Hz}$ for the wheel hop resonance.

The amplitude spectrum of the displacement excitation should be chosen such that it resembles the spectrum that the damper experiences in a vehicle. The amplitude spectrum of the excitation that the damper experiences is approximated by a linear quarter car model and a classical road profile with a first order roll-off characteristic. The parameters of the quarter car model are chosen such the quarter car approximates the behaviour of a front corner of the test vehicle to be modelled [3]. The quarter car model includes the resonances of wheel hop and body heave, but neglects the roll and pitch resonances of the vehicle body. However, the quarter car model has proven to be a sufficiently accurate 
approximation of the vertical vehicle dynamics in many applications [21].

The current excitation, which varies between $i^{-}=0.3 A$ and $i^{+}=1.6 \mathrm{~A}$, is also a multisine signal with a maximum frequency of $100 \mathrm{~Hz}$ and has the same period as the displacement excitation. The spectrum of this signal corresponds to that of the control signal generated by the model-free suspension system controller [3]: it is flat up to $10 \mathrm{~Hz}$ and has a first order characteristic at higher frequencies.

As will be shown in the following paragraph, the phases of the multisines determine the spread of the samples over the range of the excitation. A common way to select the phases of the multisines (10) is to use a realization of an independent, uniformly distributed random process on $[0,2 \pi[$. Figure 8 shows the distribution of the samples over a 10 -by10 grid on the achievable input space. These random phase multisines lead to a Gaussian distribution of the samples over the two-dimensional input space which is harmful for the model accuracy at the borders of the operating range.

Figure 9 compares the response of the damper to the random phase multisine

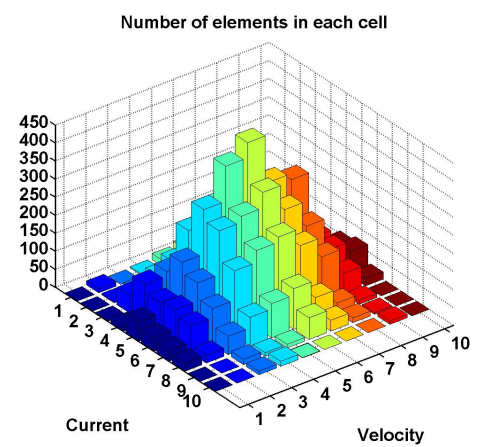

Figure 8: Distribution of the samples of random phase multisine excitation over the input space.

excitation with the response obtained by applying respectively constant minimum and maximum current excitation. This figure clearly illustrates the poor coverage of the borders of the achievable working range: for the multisine excitation, the generated damper force hardly reaches the limits.

In the following, a procedure is proposed to optimize the spread op the samples of these multisine excitations by altering the phases. The experiment design method uses the One Dimensional Homogenisation procedure developed by S. Duym [10] to design an optimal excitation for the identification of a static forcestate map of a passive damper. S. Duym considered a two-dimensional input space consisting of the damper displacement and velocity or velocity and acceleration, which are coupled variables. Neglecting this kinematic coupling in the experiment design yields measurement data that is not uniformly spread over the achievable working range of the damper. For the identification of the semiactive damper model, a two-dimensional input space of independent variables, 


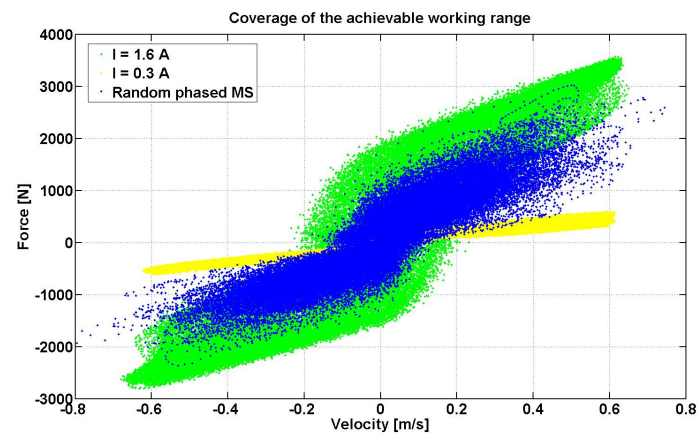

Figure 9: Coverage of the achievable working range by random phased multisine excitation.

the damper velocity and the current, is considered. Hence, the One Dimensional Homogenisation is used here on both variables independently and yields a uniform coverage of the achievable working range. In section 5.1, it is shown that the models that result from a single optimal experiment are accurate over the entire working range of the damper.

The basic idea of the One Dimensional Homogenisation is to alter the phases such that the inverse cumulative density function $(C D F)$ is linear. The inverse cumulative density function returns the value below which the realizations of the process would fall with a probability $p$. For the desired uniform distribution, the inverse $\mathrm{CDF}$ is linear .

The procedure starts from random phase multisine signals for the velocity and current calculated by using equation 10 . The steps of the iterative algorithm are explained in the following for the velocity signal. They are analogously applied to the current.

1. Sort the velocity signal $\left\{\dot{x}_{k}\right\}$ in ascending order, obtaining the set $\left\{\dot{y}_{k}\right\}$. This ordering can be represented by following transformation:

$$
\left\{\dot{y}_{k}\right\}=T\left\{\dot{x}_{k}\right\}
$$

where $T$ is a permutation matrix and $k$ is an index, ranging from 0 to $N$, the number of samples in the data set.

2. The inverse CDF of the velocity signal $\left\{\dot{x}_{k}\right\}$ can easily be calculated by considering $\dot{y}_{k}$ as a function of its normalized indices $\left\{\frac{1}{N}, \frac{2}{N}, \ldots, \frac{i}{N}, \ldots, \frac{N}{N}\right\}$.

3. The desired uniform excitation has a linear inverse CDF. A set of samples $\left\{\dot{z}_{k}\right\}$ can be calculated from this known ideal inverse CDF:

$$
\begin{aligned}
\left\{\dot{z}_{k}\right\}= & \left\{-\frac{N-1}{2},-\frac{N-3}{2}, \ldots,-\frac{2 k-N-1}{2}, \ldots\right. \\
& \left.\frac{N-3}{2}, \frac{N-1}{2}\right\} \frac{2 \sqrt{3} \Sigma_{\dot{x}_{r}}}{N-1}
\end{aligned}
$$


The factor $\sqrt{3}$ is introduced to give the signal the proper scaling. $\Sigma_{\dot{x}_{r}}$ represents the root mean square values of the velocity signal $\dot{x}_{r} .\left\{\dot{z}_{k}\right\}$ is a set of equidistant points which are sorted in ascending order.

4. A new velocity signal can be obtained by applying the inverse of the sorting operation (11) to $\left\{\dot{z}_{k}\right\}$ :

$$
\left\{\dot{w}_{k}\right\}=T^{-1}\left\{\dot{z}_{k}\right\}
$$

5. Calculate the discrete fourier transformation $\{\dot{W}(s)\}$ of the signal $\left\{\dot{w}_{k}\right\}$. Finally, the new velocity signal is obtained by calculating the inverse fourier transformation of the sequence obtained by combining the phases of the signal $\left\{\dot{W}_{k}\right\}$ and the original amplitude spectrum of $\left\{\dot{x}_{k}\right\}$.

6. Scale the velocity signal and corresponding displacement signal such that it does not violate the constraints of the damper.

7. Apply steps 1 to 6 to the current signal.

8. Evaluate the coverage of the obtained excitation. The steps 1 to 7 are repeated until the coverage of the working range is sufficiently uniform or a specified maximum number of iterations is reached.

To evaluate the coverage of the achievable working range, a criterion has to be defined that expresses the difference between the desired and the realized distribution of the samples. The distribution of the samples can be evaluated by counting the number of samples in each cell of a equidistant rectangular grid over the achievable working range. For the desired uniform distribution this is:

$$
n_{\text {ideal }}=\frac{N}{n_{v} n_{i}}
$$

where $n_{\text {ideal }}$ represents the number of samples per cell, $N$ the number of samples in the data set and $n_{v}$ and $n_{i}$ the number of intervals of the grid on the velocity and current axis respectively. The criterion, referred to as the coverage index $C$, can then be defined as:

$$
C=\kappa \sum_{p=1}^{n_{v}} \sum_{q=1}^{n_{i}}\left(n_{p q}-n_{\text {ideal }}\right)^{2} .
$$

$\kappa$ is a scaling factor:

$$
\kappa=\frac{1}{N\left(1-\frac{1}{n_{v} n_{i}}\right)},
$$

which is chosen such that the criterion is independent of the number of cell in the grid and of the total number of samples in the data set. More details and background about this experiment design technique can be found in [11].

Figure 10 shows a bar diagram of the number of samples in each cell of a 10 by 10 rectangular grid for an optimized multisine excitation. In figure 11 the response of the damper is compared to the minimal and maximal achievable damper force. This figure clearly illustrate the effectiveness of the approach: the samples are distributed more uniformly over the achievable working range in 
comparison with random phased excitation (see figure 9) The coverage index for this optimized excitation amounts 5.6, while this of the random phase excitation shown in figure 9 , is 833.5 .

The experiment design procedure optimizes the damper velocity. This signal is

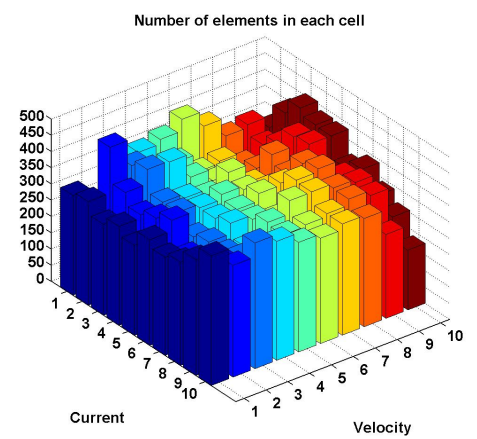

Figure 10: Distribution of the samples of the optimized multisine excitation over the input space.

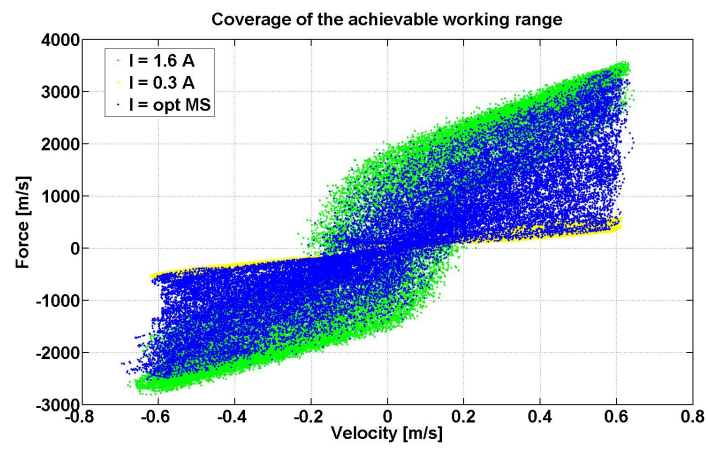

Figure 11: Coverage of the achievable working range by optimized multisine excitation.

converted to a corresponding displacement signal, which can then be applied to the hydraulic test rig. This conversion is performed in frequency domain with a method analogue to the derivation used to calculate the damper velocity and acceleration from the damper displacement (see section 4.1) and is exact, since the designed signal is periodic.

\section{Experimental validation}

This section discusses the experimental validation of the optimal experiment design method (section 5.1) and the proposed identification procedure (section 
5.2) using measurement data obtained with the test setup described in section 4.1 .

\subsection{Evaluation of the experiment design method}

Since the response of the semi-active damper is expected to be dependent of the excitation level, measurements at five different excitation levels are performed:

- $M$ 1: maximum velocity $0.2 \mathrm{~m} / \mathrm{s}$

- $M$ 2: maximum velocity $0.4 \mathrm{~m} / \mathrm{s}$

- $M$ 3: maximum velocity $0.6 \mathrm{~m} / \mathrm{s}$

- $M$ 4: maximum velocity $0.8 \mathrm{~m} / \mathrm{s}$

- M 5: maximum velocity $1.0 \mathrm{~m} / \mathrm{s}$

The excitation signals for these measurements have been calculated with the optimal experiment design method described in section 4.2. The identification procedure discussed in section 3, is applied to the data set of the medium level excitation $M$ 3. The initial regression vector, $\varphi_{i n i}$, consists of three elements:

$$
\varphi_{\text {ini }}\left(t_{n}\right)=\left[\begin{array}{lll}
\widehat{F}\left(t_{n-1}\right) & v_{r}\left(t_{n-1}\right) & i\left(t_{n-1}\right)
\end{array}\right] .
$$

The algorithm was allowed to extend the regression vector with the delayed values of damper displacement $x_{r}$, velocity $v_{r}$, acceleration $a_{r}$, current $i$ and the simulated damper force $\widehat{F}$. These variables have been selected based on models of passive and MR-dampers published in literature (see section 2.3) and physical insights in the semi-active damper dynamics. By including the damper force, feedback is introduced in the model which helps to limit the number regression variables. The velocity and acceleration are related to the oil flow dynamics and the displacement to the accumulator filling (see section 2).

The maximum delay of the allowed regressors was limited to 5 samples. The used neural network has one hidden layer of 14 nodes with sigmoid activation functions and a linear output layer. The number of nodes in the hidden layer has been determined by trial and error and is a compromise between model accuracy and complexity. For the parameter estimation, the first 20000 samples of the data set were used, while the remaining 12768 samples were used for the model evaluation.

The identification algorithm returns a model with following optimal regression vector:

$$
\begin{aligned}
& \varphi_{M 3}\left(t_{n}\right)=\left[\begin{array}{lllll}
\widehat{F}\left(t_{n-1}\right) & \widehat{F}\left(t_{n-2}\right) & \widehat{F}\left(t_{n-3}\right) & x_{r}\left(t_{n-1}\right) & v_{r}\left(t_{n-1}\right)
\end{array} \ldots\right. \\
& \left.v_{r}\left(t_{n-2}\right) \quad a_{r}\left(t_{n-2}\right) \quad i\left(t_{n-2}\right)\right]^{T} .
\end{aligned}
$$

The iterative identification procedure stopped when the method could not find an additional regressor that improved the model accuracy (see section 3.2). The execution of the identification procedure took about 12 hours on a PC equipped with a $3 \mathrm{GHz}$ processor. Nine iteration steps were performed, during which 57 
different models were estimated and evaluated. In the following, we will refer to the model identified using the data set $M 3$ as $N N O E_{O P H}$. To quantify the accuracy of the obtained simulation model, the relative root mean square simulation error (rrmsse) is used:

$$
\text { rrmsse }=\frac{\sqrt{\sum_{n=1}^{N}\left[F\left(t_{n}\right)-\widehat{F}\left(t_{n}\right)\right]^{2}}}{\sqrt{\sum_{n=1}^{N} y\left(t_{n}\right)^{2}}} \cdot 100 \% .
$$

Figure 12 shows the simulated damper force and compares it to the measured damper force for a part of the validation set of measurement $M$ 3. The rrmssevalue of this simulation is about $4.0 \%$.

To evaluate the effectiveness of the optimal experiment design, the simulation

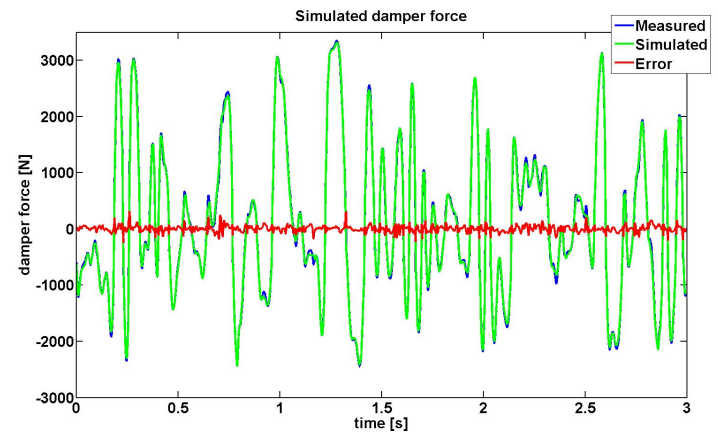

Figure 12: Damper force simulated with the identified $N N O E$-model based on the optimized excitation data set.

accuracy of the model $N N O E_{O P H}$ will be compared to a model identified using the data set shown in figures 8 and 9 , which is a random phase multisine excitation that is not optimized and that has an excitation level that corresponds to that of data set $M 3$. In the following, this later model will be denoted as $N N O E_{R P H}$.

Table 1 shows the rrmss-error of the models $N N O E_{O P H}$ and $N N O E_{R P H}$ obtained using the data sets $M 1$ to $M 5$. The table clearly demonstrates that the $N N O E_{O P H}$ model is far more accurate than the $N N O E_{R P H}$ model. Not only for medium level excitation but also for excitations at lower and higher amplitudes. Only at very low amplitude level, the model appears to be less accurate. The semi-active damper exhibits a significantly different behaviour at low damper velocities, which are dominant in data set $M 1$, due the nonlinear flow characteristics of the damper valves [4].

\subsection{Evaluation of the regression vector selection procedure}

To evaluate the regression vector found by the identification procedure, the accuracy of the $N N O E_{O P H}$ model with regression vector $\varphi_{M 3}$ (see equation 


\begin{tabular}{|l|c|c|c|c|c|}
\hline & $M 1$ & $M 2$ & $M 3$ & $M 4$ & $M 5$ \\
\hline$N N O E_{O P H}$ & $8.4 \%$ & $4.8 \%$ & $3.8 \%$ & $5.6 \%$ & $5.9 \%$ \\
$N N O E_{R P H}$ & $8.6 \%$ & $7.6 \%$ & $9.4 \%$ & $12.1 \%$ & $12.3 \%$ \\
\hline
\end{tabular}

Table 1: Comparison of the simulation performance of the $N N O E_{O P H}$ and $N N O E_{R P H}$ models for measurements at different excitation levels

17) is compared to that of the models obtained by omitting all samples of the model output, $\widehat{F}$, or of one of the input variables $x_{r}, v_{r}, a_{r}$ or $i$ from the regression vector. The optimal and reduced regression vectors considered in this comparison are:

$$
\begin{aligned}
& \varphi_{M 3}\left(t_{n}\right) \quad: \quad \text { see equation } 17 \\
& \varphi_{1}\left(t_{n}\right)=\left[\begin{array}{lllll}
x_{r}\left(t_{n-1}\right) & v_{r}\left(t_{n-1}\right) & v_{r}\left(t_{n-2}\right) & a_{r}\left(t_{n-2}\right) & i\left(t_{n-2}\right)
\end{array}\right]^{T}, \\
& \varphi_{2}\left(t_{n}\right)=\left[\begin{array}{lllll}
\widehat{F}\left(t_{n-1}\right) & \widehat{F}\left(t_{n-2}\right) & \widehat{F}\left(t_{n-3}\right) & v_{r}\left(t_{n-1}\right) & v_{r}\left(t_{n-2}\right)
\end{array} \ldots\right. \\
& \left.a_{r}\left(t_{n-2}\right) \quad i\left(t_{n-2}\right)\right]^{T}, \\
& \varphi_{3}\left(t_{n}\right)=\left[\begin{array}{llllll}
\widehat{F}\left(t_{n-1}\right) & \widehat{F}\left(t_{n-2}\right) & \widehat{F}\left(t_{n-3}\right) & x_{r}\left(t_{n-1}\right) & a_{r}\left(t_{n-1}\right) & i\left(t_{n-2}\right)
\end{array}\right]^{T}, \\
& \varphi_{4}\left(t_{n}\right)=\left[\begin{array}{lllll}
\widehat{F}\left(t_{n-1}\right) & \widehat{F}\left(t_{n-2}\right) & \widehat{F}\left(t_{n-3}\right) & x_{r}\left(t_{n-1}\right) & v_{r}\left(t_{n-1}\right)
\end{array} \ldots\right. \\
& \left.v_{r}\left(t_{n-2}\right) \quad i\left(t_{n-2}\right)\right]^{T} \text {, } \\
& \varphi_{5}\left(t_{n}\right)=\left[\begin{array}{lllll}
\widehat{F}\left(t_{n-1}\right) & \widehat{F}\left(t_{n-2}\right) & \widehat{F}\left(t_{n-3}\right) & x_{r}\left(t_{n-1}\right) & v_{r}\left(t_{n-1}\right)
\end{array} \ldots\right. \\
& \left.v_{r}\left(t_{n-2}\right) \quad a_{r}\left(t_{n-2}\right)\right]^{T} \text {. }
\end{aligned}
$$

Table 2 summarizes the rrmsse-values for the different models. It is obvious that the omission of the model output variables $\left(\varphi_{1}\right)$, the damper velocity $\left(\varphi_{3}\right)$ and the current $\left(\varphi_{5}\right)$ have the largest influence on the model accuracy.

To conclude the evaluation, the proposed identification procedure is applied to

\begin{tabular}{|c|c|c|c|c|c|c|}
\hline & $\varphi_{M 3}$ & $\varphi_{1}$ & $\varphi_{2}$ & $\varphi_{3}$ & $\varphi_{4}$ & $\varphi_{5}$ \\
\hline rrmsse & $3.8 \%$ & $8.6 \%$ & $4.3 \%$ & $43 \%$ & $4.2 \%$ & $38.7 \%$ \\
\hline
\end{tabular}

Table 2: Rrmsse-values obtained for the models with the different regression vectors

the data sets $M 1$ to $M 5$. The regression vectors returned by the procedure are the following:

$$
\begin{aligned}
& \varphi_{M 1}\left(t_{n}\right)=\left[\begin{array}{lllll}
\widehat{F}\left(t_{n-1}\right) & \widehat{F}\left(t_{n-2}\right) & v_{r}\left(t_{n-1}\right) & a_{r}\left(t_{n-1}\right) & a_{r}\left(t_{n-2}\right)
\end{array} \ldots\right. \\
& \left.i\left(t_{n-1}\right)\right]^{T} \text {. } \\
& \varphi_{M 2}\left(t_{n}\right)=\left[\begin{array}{lllll}
\widehat{F}\left(t_{n-1}\right) & \widehat{F}\left(t_{n-2}\right) & \widehat{F}\left(t_{n-3}\right) & x_{r}\left(t_{n-1}\right) & v_{r}\left(t_{n-1}\right)
\end{array} \ldots\right.
\end{aligned}
$$

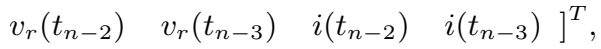

$$
\begin{aligned}
& \varphi_{M 3}\left(t_{n}\right)=\left[\begin{array}{lllll}
\widehat{F}\left(t_{n-1}\right) & \widehat{F}\left(t_{n-2}\right) & \widehat{F}\left(t_{n-3}\right) & x_{r}\left(t_{n-1}\right) & v_{r}\left(t_{n-1}\right)
\end{array} \ldots\right. \\
& \left.v_{r}\left(t_{n-2}\right) \quad a_{r}\left(t_{n-2}\right) \quad i\left(t_{n-2}\right)\right]^{T}, \\
& \varphi_{M 4}\left(t_{n}\right)=\left[\begin{array}{lllll}
\widehat{F}\left(t_{n-1}\right) & \widehat{F}\left(t_{n-2}\right) & \widehat{F}\left(t_{n-3}\right) & x_{r}\left(t_{n-1}\right) & v_{r}\left(t_{n-2}\right)
\end{array} \ldots\right.
\end{aligned}
$$




$$
\begin{aligned}
& \left.v_{r}\left(t_{n-3}\right) \quad i\left(t_{n-1}\right) \quad i\left(t_{n-2}\right)\right]^{T}, \\
& \varphi_{M 5}\left(t_{n}\right)=\left[\begin{array}{lllll}
\widehat{F}\left(t_{n-1}\right) & \widehat{F}\left(t_{n-2}\right) & x_{r}\left(t_{n-1}\right) & v_{r}\left(t_{n-1}\right) & a_{r}\left(t_{n-1}\right)
\end{array} \ldots\right.
\end{aligned}
$$

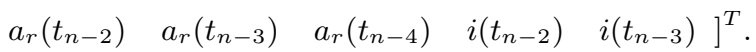

The obtained regression vectors show both similarities and differences. They all include two or three samples of the simulated damper force, some samples of the velocity and one or two samples of the current. For the damper displacement and acceleration, there is less uniformity. Since these are black-box models, it is difficult to give a physical interpretation to the obtained regression vectors. Finally, the identified models are validated using all five data sets. The results are summarized in table 3 and illustrate that the model identified using the low level excitation does not describe the dynamic damper behaviour accurately at higher level excitations, which can be attributed to two different causes. Firstly, the damper dynamics depend strongly on the velocity: at low level excitations, the oil flow dynamics exhibit a different behaviour then during medium to high level excitations [4]. Secondly, it is a general known problem that neural networks yield poor accuracy when evaluating them outside the range of data set used for the identification of the model parameters. However, the models $N N O E_{M 3}$ and $N N O E_{M 4}$ describe the damper behaviour fairly well over the entire working range. Hence, the extrapolation problem is believed to be of minor importance and the poor results of models $N N O E_{M 1}$ and $N N O E_{M 2}$ can be mainly attributed to the difference in oil flow dynamics. This motivates a proper design of the excitation signals, taking the intended application of the

\begin{tabular}{|c|c|c|c|c|c|c|}
\hline & \multicolumn{5}{|c|}{ Model } \\
\hline & & $N N O E_{M 1}$ & $N N O E_{M 2}$ & $N N O E_{M 3}$ & $N N O E_{M 4}$ & $N N O E_{M 5}$ \\
\hline \multirow{5}{*}{ Data } & $M 1$ & $4.5 \%$ & $5.9 \%$ & $8.4 \%$ & $9.8 \%$ & $9.4 \%$ \\
\hline & $M 2$ & $10.7 \%$ & $4.0 \%$ & $4.8 \%$ & $5.6 \%$ & $5.9 \%$ \\
\hline & M3 & $15.8 \%$ & $8.3 \%$ & $3.8 \%$ & $4.4 \%$ & $4.4 \%$ \\
\hline & M4 & $22.4 \%$ & $12.9 \%$ & $5.6 \%$ & $4.1 \%$ & $4.4 \%$ \\
\hline & $M 5$ & $21.1 \%$ & $15.4 \%$ & $5.9 \%$ & $4.8 \%$ & $4.7 \%$ \\
\hline
\end{tabular}
model into account.

Table 3: Rrmsse-values obtained with the models identified using the data sets $M 1$ tot $M 5$

\section{Conclusion}

This paper presented an identification procedure for a nonlinear black-box model of an electro-hydraulic, continuously variable, semi-active damper for a passenger car. The procedure includes a method to design optimal excitations. The semi-active damper has two independent inputs being the displacement velocity and the current applied to the cvsa-valve, which form a two-dimensional input space. For the excitation, periodic multisine signals are used of which the amplitude spectra are chosen such that they approximate well the excitation the damper experiences in a vehicle when driving over a classical road profile. The 
phases of the multisine signals are optimized such that a uniform distribution of the samples over the achievable working range is obtained.

The black-box model consists of a neural network with one hidden layer of sigmoidal neurons and a linear output neuron for mapping the regression vector to the simulated damper force. The identification procedure includes an iterative method to optimize of the regression element vector. The resulting regression vector includes damper displacement, velocity, acceleration, current and the simulated damper force. These variables are available in a multi-body simulation environment, such that the proposed model can be easily incorporated in a full-vehicle simulation model. The identified damper model is able to simulate the measured damper forces accurately, up to a error margin of $4 \%$, except at very low damper velocity.

The effectiveness of the proposed optimal experiment design procedure is validated by comparing a model identified using a data set measured with the optimized excitation to a model identified using a data set measured with the classical random phase multisine excitation. It is demonstrated that a model which is identified using a single optimal experiment, can accurately describe the dynamic behaviour of the damper over its entire working range.

Future research will focus on a way to adopt the model in order to include an accurate description of the damper dynamics at low excitation level.

\section{Acknowledgements}

This work is a the result of a cooperation between Tenneco Automotive Europe and the department of the mechanical engineering of K.U.Leuven. The financial support of the Institute for the Promotion of Innovation by Science and Technology in Flanders (IWT) is gratefully acknowledged.

This work also benefits from K.U.Leuven-BOF EF/05/006 Center-of-Excellence Optimization in Engineering and from the Belgian Programme on Interuniversity Attraction Poles, initiated by the Belgian Federal Science Policy Office.

\section{References}

[1] A. Nilsson, K. Reybrouck, Semi-active suspension systems, $2^{\text {nd }}$ Advanced Suspension Systems, Frankfurt, Germany, 2007.

[2] M. Harrison, Vehicle Refinement: Controlling Noise and Vibration in Road Vehicles, Elsevier Butterworth-Heinemann, Oxford, UK, 2004.

[3] J. Swevers, C. Lauwerys, B. Vandersmissen, M. Maes, K. Reybrouck, P. Sas, A model-free control structure for the on-line tuning of the semi-active suspension of a passenger car, Mechanical Systems and Signal Processing, vol. 21 (3), pp. 1422-1436, 2007.

[4] S.W.R. Duym, Simulation Tools, Modelling and identification, for an Automotive Shock Absorber in the Context of Vehicle Dynamics, Vehicle System Dynamics, vol. 33 (4), pp. 261-285, 2000. 
[5] A. Patel, J.F. Dunne, NARX Neural Network modelling of Hydraulic Suspension Dampers for Steady-state and Variable Temperature Operation, Vehicle System Dynamics, vol 40 (5), pages 285-328, 2003.

[6] A. Leva, L. Piroddi, NARX-based technique for the modelling of magnetorheological damping devices, Smart Materials and Structures, vol. 11 (1), pp. 79-88, 2002.

[7] S.M. Savaresi, S. Bittanti, M. Montiglio, Identification of semi-physical and black-box non-linear models: the case of MR-dampers for vehicles control, Automatica, vol. 41, pages 113-127, 2005.

[8] L. Piroddi, W. Spinelli, An identification algorithm for polynomial NARX models based on simulation error minimization, Int. Journal of Control, vol. 76 (17), pp. 1767-1781, 2003.

[9] M. Witters, J. Swevers, Black-box identification of a continuously variable semi-active damper, Proc. of the IEEE Multi-Conference on Systems and Control, San Antonio, Texas, USA, pages 19-24, 2008.

[10] S.W.R Duym, J. Schoukens, Design of excitation signals for the restoring force surface method, Mechanical Systems and Signal Processing, vol. 9 (2), pp. 139-158, 1995.

[11] S.W.R Duym, Nonparametric Identification of Nonlinear Mechanical Systems, PhD V.U.B., Dept. of Electrical Engineering, Brussel, 1998.

[12] B.F. Spencer Jr., S.J. Dyke, M.K. Sain, J.D. Carlson, Phenomenological Model of a Magnetorheological Damper, ASCE Journal of Engineering Mechanics, vol. 123, pp. 230-238, 1997.

[13] Y.K. Wen, Method of Random Vibration of Hysteretic Systems, ASCE Journal of Engineering Mechanics, Vol. 102, pp. 249263, 1976.

[14] M. Nørgaard, O. Ravn, N.K. Poulsen, L.K. Hansen, Neural networks for Modelling and Control of Dynamic Systems, Springer-Verlag, London, UK, 2000.

[15] A.J. Barber, Accurate Models for Bushings and Dampers using the Empirirical Dynamics Method, $14^{\text {th }}$ European ADAMS Users' Conference, Berlin, Germany, 1999.

[16] J. Sjöberg, Q. Zhang, L. Ljung, A. Benveniste, B. Delyon, P-Y. Glorennec, H. Hjalmarsson, A. Juditsky, Nonlinear Black-Box Modeling in System identification: a Unified Overview, Automatica, vol. 31 (12), pp. 1691 1724, 1995.

[17] G. Cybenco, Approximation by Superpositions of Sigmoidal Functions, Mathematics of Control, Signals, and Systems, vol. 2 (4), pp. 303-314, 1989. 
[18] M. Nørgaard, Neural Network Based System Identification Toolbox, Tech. Report. 00-E-891, Department of Automation, Technical University of Denmark, 2000.

[19] J. Nocedal, S.J. Wright, Numerical Optimization, Springer-Verlag, New York, 1999.

[20] R. Pintelon, J. Schoukens, System Identification: A Frequency Domain Approach, IEEE Press, New York, 2001.

[21] D. Hrovat, Survey of Advanced Suspension Developments and Related Optimal Control Applications, Automatica, vol. 33 (10), pp. 1781-1817, 1997. 